\title{
1. Introduction to the Clean Development Mechanism
}

We are not only losing land but also agricultural productivity due to frequent salt water incursion which has affected five square kilometres of our land since 1969 . . . Either our island is sinking or the sea is rising.

Jalaluddin Saha, India, in WWF (2007)

[T] limit the temperature increase to $2^{\circ} \mathrm{C}$ above pre-industrial levels, developed countries would need to reduce emissions in 2020 by $10-40 \%$ below 1990 levels and in 2050 by approximately $40-95 \%$. Emissions in developing countries would need to deviate below their current path by $2020 \ldots$

Gupta et al. (2007a, p. 748)

The natural next step in the evolution of the Kyoto Protocol, and the climate change regime as a whole, is to create clear opportunities and financial incentives for developing country Parties to participate in international emissions trading if they so choose.

AOSIS (2011, p. 43)

In the past two decades, man-made climate change has become increasingly recognized, both scientifically and politically, as a real and urgent threat to humanity and a relevant consideration in shaping future development paths (Parry et al. 2007, pp.44-7; Solomon et al. 2007, pp. 24-32). It has thus emerged as a rapidly growing international policy arena. Due to the far-reaching implications of the actions needed to tackle the climate change threat, enormous challenges subsist in the political sphere for reaching agreements on how, when and where to act.

Tackling climate change implies several types of complementary responses. Mitigation implies reducing the emissions of anthropogenic carbon dioxide $\left(\mathrm{CO}_{2}\right)$ and other greenhouse gases (GHG) to the atmosphere in order to slow down climate change and prevent it from reaching dangerous levels. It, thus, addresses the causes of climate change. Adaptation aims to reduce the already occurring adverse consequences of climate change and to enhance positive impacts. Enabling measures encompass supportive actions, particularly towards developing and vulnerable countries, which lack the means or capacity to mitigate or adapt to 
climate change on their own. They hence comprise capacity building activities, technology development and transfer and the provision of financial support. While all these types of measures are recognized as important constituents of a successful climate policy regime, the Climate Change Convention and its Kyoto Protocol have so far focused mainly on mitigation measures. The Bali Action Plan, agreed at the Conference of the Parties (COP) 13 in 2007, and the subsequent agreements in Copenhagen (COP 15, 2009), Cancún (COP 16, 2010) and Durban (COP 17, 2011) provide for a stronger focus on adaptation, financing, capacity building and technology transfer in the framework of a new international climate agreement still under negotiation. These new developments, however, are outside the scope of this book.

Signed in 1992 and ratified by 195 parties as of April 2013, the United Nations Framework Convention on Climate Change (UNFCCC) was the first international treaty agreed to promote global action towards preventing dangerous climate change. The convention has the objective of achieving a 'stabilization of greenhouse gas concentrations in the atmosphere at a level that would prevent dangerous anthropogenic interference with the climate system' (UN 1992, Art. 2). While it asks parties to report on progress towards this goal and also states the aim, for a group of countries, to return to the 1990 levels of GHG emissions, it lacks legally binding emission reduction targets for individual countries and an enforcement and compliance system. The UNFCCC places the heaviest responsibility for combating climate change on 27 industrialized countries and 12 economies in transition, which are the source of most past and current GHG emissions. Based on its principle of common but differentiated responsibilities (UN 1992, Art. 3.1), and acknowledging that economic development is vital for developing countries, the convention accepts that their emissions will continue to rise in the foreseeable years (UN 1992, chapeau). It nevertheless intends to help these countries to limit their emissions without compromising their growth.

The Kyoto Protocol, the first legally binding treaty under the convention, was adopted in 1997 and entered into force in February 2005. As of April 2013, 191 countries and the European Union are parties to the protocol. Under Article 3.1 of the Kyoto Protocol (UN 1998), industrialized countries (known as Annex I or Annex B Parties) ${ }^{1}$ agreed to reduce their aggregate GHG emissions to at least 5 per cent below 1990 levels by $2008-12 .^{2}$

From a macroeconomic perspective, mitigation implies adopting low greenhouse-gas processes and technologies, ideally without slowing growth and development. In general it is accepted that mitigation measures are more expensive in industrialized countries, where efficient and 
state-of-the-art technologies are more common (Stern 2007, pp. 245-6). Furthermore, as climate change is a global problem, in terms of the environmental outcome it does not matter where the emission reductions take place, but how much is reduced overall. Hence, to facilitate that Annex I countries achieve their emission reduction targets in a cost-effective manner, the Kyoto Protocol established three market-based flexible mechanisms: International Emissions Trading, Joint Implementation (JI) and the Clean Development Mechanism (CDM). The first mechanism allows for trading of emissions allowances between countries with reduction targets, while JI refers to investment in emission reduction projects in other Annex I countries, and the CDM allows for emission reduction or sequestration projects in developing countries without emission targets (non-Annex I countries). CDM projects generate certified emission reduction credits (CERs) ${ }^{3}$ that can be acquired by Annex I parties and used for offsetting emissions in excess of their targets. Because CERs are used for offsetting (or compensating for) emissions made in Annex I countries, the CDM does not lead to more emission reductions, but only to a reduction in the cost of achieving the Annex I targets.

The CDM is thus the only Kyoto Protocol instrument that involves the participation of developing countries in climate change mitigation. It has the double aim of ensuring cost-effectiveness of mitigation measures, while at the same time assisting 'Parties not included in Annex I in achieving sustainable development and in contributing to the ultimate objective of the Convention' (UN 1998, Art. 12.2). The need to ensure that CDM projects have an equitable geographic distribution across host developing countries emerged as a condition for the CDM's second aim to be fulfilled (UNFCCC 2001b, p. 20).

In order to ensure that the CDM fulfils its double aim, a complex project cycle and governance system was established (Figure 1.1). Proposed CDM projects first need to be approved by the designated national authority (DNA) of the country in which the project is located, which establishes the necessary criteria for the project to contribute to the country's sustainable development priorities. A standardized project design document (PDD) describing the project's aims, baseline calculation, ${ }^{4}$ additionality determination, ${ }^{5}$ estimated emission reductions, monitoring plan and environmental impacts needs to be submitted to an independent auditor (the designated operational entity or DOE), who validates that the project fulfils all the requirements for registration. Once the project has been validated, its registration by the CDM Executive Board (CDM EB) is requested. ${ }^{6}$ If three or more members of the CDM EB consider that some of the CDM requirements are not clearly substantiated, the project is subject to a review, otherwise it is registered. A review may result in 
Project participant with CDM EB-approved baseline and monitoring methodology

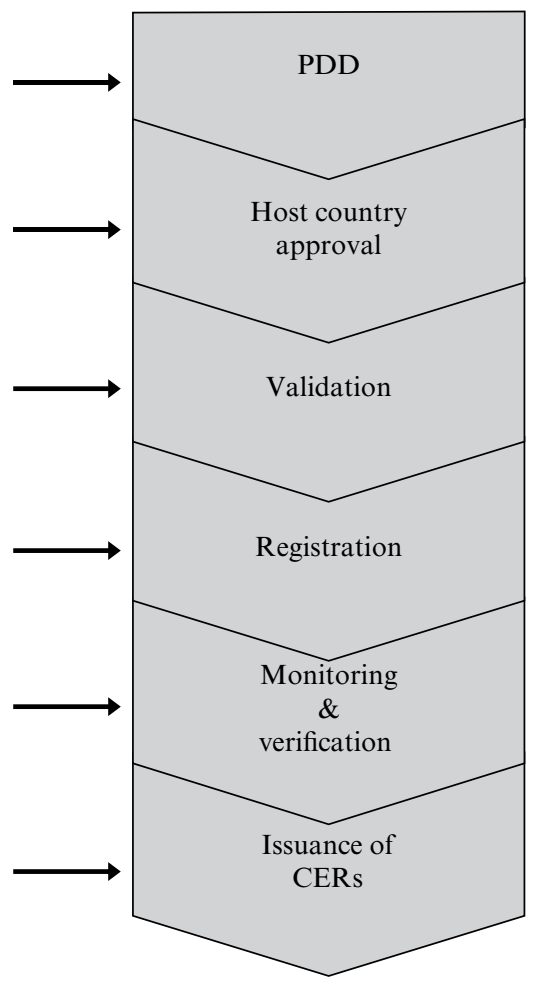

Designated Operational Entity's Validation Report

Validation failure

UNFCCC Secretariat: Completeness Check CDM EB: (Review) and Registration

| Review and rejection

Emissions monitored by project participant DOE's Verification Report

UNFCCC Secretariat: Completeness Check CDM EB: (Review) and Issuance

Review and rejection at issuance

\section{Source: Adapted from UNFCCC (n.d.)}

\section{Figure 1.1 The CDM's project cycle}

requests for corrections; once these are fulfilled the project is registered, otherwise it is rejected. Once a project is registered, its emission reductions need to be monitored continuously and reported each year. A DOE verifies the monitoring report, and if everything is correct, the CDM EB issues the corresponding CERs. Here again, if the CDM EB detects any problems in the verification of the monitoring report, it may request a review of the proposed issuance of CERs (UNFCCC 2005a).

\subsection{THE CDM: RISE AND FALL OF AN UNEXPECTED GIANT?}

At first sight, the statistics about the CDM depict the large success of this mechanism so far: as of April 2013, 6660 CDM projects have been 
registered in 85 countries, and are expected to reduce about 860 million tonnes equivalent carbon dioxide $\left(\mathrm{tCO}_{2} \mathrm{e}\right)$ emissions per year; another 2337 projects still in the process of validation or registration could add about 385 million $\mathrm{tCO}_{2}$ e annual reductions if they are successful (UNEP Risoe Centre 2013a). Such numbers quite exceed the initial expectations on this mechanism. At the beginning of the 2000s, experts of the Kyoto process and the nascent carbon market rather expected a larger role of international emissions trading and $\mathrm{JI}$ in economies in transition, which had a large surplus of carbon emission allowances inherited from their economic downturn in the 1990s. In contrast, the CDM would be slowed down by high transaction costs and by the institutional, information and infrastructural barriers existing in developing countries (Michaelowa and Jotzo 2005).

Substantial criticism has nonetheless overshadowed this initially unexpected success over the years. First, CDM projects are highly concentrated in a few advanced developing countries. China, India and Brazil by themselves host over 5000 of the registered projects. While this geographical distribution closely mirrors the available emission reduction potential and the investment conditions in the host countries (Flues 2010; Lütken 2011; Winkelman and Moore 2011; Dolšak and Crandall 2013), it is perceived by some parties as unequal (see, for example, Government of Sri Lanka 2008 , p. 24), particularly in view of the benefits the CDM is expected to generate for the host countries: besides financial flows for emission reductions, technology transfer and sustainable development benefits. The CDM is overall a success in numbers, but the complexity of its project cycle and the high transaction costs that it entails make it comparatively more difficult for poor countries and small projects to access the mechanism (Ellis and Kamel 2007).

Second, many CDM projects have not brought about the expected sustainable development benefits, and in some cases there are substantial doubts about their social and environmental integrity (Borges da Cunha et al. 2007; Lenzen et al. 2007; Rudolph 2007; Sirohi 2007; Cole and Roberts 2011). While several schemes about how to effectively assess the sustainable development contribution of CDM projects have been proposed in the literature (for example, Sutter and Parreño 2007; Olsen and Fenhann 2008), due to sovereignty concerns it is the prerogative of the host countries to decide upon and assess such sustainability criteria. Information on how many project proposals are rejected by host country DNAs because they fail to satisfy host country requirements is not publicly available. However, as Schneider (2007, p.46) points out, 'Generally, it can not be observed that host countries prioritize projects with high sustainable development impacts by rejecting projects with little or no sustainable development impact.' 
Third, the quality of the achieved emission reductions - in terms of being 'real' and 'additional' - has repeatedly been put into question (Michaelowa and Purohit 2007; Schneider 2007; Castro and Michaelowa 2008; Haya 2009). According to interviews performed with some project developers in late 2007, even project developers themselves have doubts about the additionality of some projects, especially very large ones, as they involve very high levels of investment and it is easy to tweak numbers and choose indicators so that they can show that the project would not have been financially attractive in the absence of the CDM (Castro and Michaelowa 2008). If emission reductions from CDM projects are not real but just artefacts of accounting tricks, then the mechanism is actually leading to an increase in emissions to the atmosphere.

Fourth, the CDM, relying on the market, focuses mostly on the cheap emission reduction options, leading to high rents and windfall profits for project developers (Wara 2008), and/or to the fear that selling the cheap emission reduction opportunities now will make mitigation in developing countries more expensive later (World Bank 2003, pp.31-2), potentially leading to disincentives for developing countries to engage in other types of domestic mitigation action.

Finally, given the size of the mitigation challenge, not only industrialized countries need to have more ambitious emission reduction targets, but also developing countries need to contribute to reductions beyond offsetting (Gupta et al. 2007a; Garnaut 2012). Demands for reforming the CDM, or creating other market mechanisms, so that corresponding incentives for developing countries are created, have become louder since Copenhagen, and the academic discussion has also embraced proposals on how to reform the CDM so that it can contribute to emission reductions beyond offsetting (see, for example, Schneider 2009).

But another - even greater - challenge has emerged for the CDM. After peaking at a level of 20.9 euros in June 2008, the price of CER credits from CDM projects has unstoppably spiralled downwards, collapsing to just one cent in April 2013. A mix of circumstances and political failures has led to a strongly oversupplied carbon market with little hope for a quick recovery. During the international climate negotiations at Copenhagen, Cancún and Durban, parties to the UNFCCC failed to reach a successful agreement with ambitious emission reduction targets for developed countries and other major emitters for the period after 2012. Instead, they settled on a system of low-ambition national emissions pledges, along with a second commitment period under the Kyoto Protocol that no longer includes emission reduction commitments for Canada, Japan, New Zealand and the Russian Federation (UNFCCC 2012b). This has given place to a strongly reduced demand market for CDM credits. In addition, the global eco- 
nomic crisis that started in 2008 led also to lower demand for carbon allowances and credits from European firms than foreseen under the European Union Emissions Trading System (EU ETS), which has historically been the largest (and is almost the only remaining) source of demand for CERs. Lack of political will by European governments to address the oversupply of emission allowances in the EU ETS is fuelled by the domestic economic downturn and the watered-down international regime.

At the same time as demand was dwindling, supply of carbon credits has been rising. In a move intended to reduce the use of offsetting mechanisms and encourage more meaningful contribution to emission reductions by developing countries, the EU unilaterally imposed restrictions on the types of CERs that would be accepted into the EU ETS from 2013 onwards (see Chapter 3 for more details). This led to a rush by CDM project developers to register as many projects as possible before the deadline set by the EU (31 December 2012), exacerbating the market imbalance even more. Similarly, new rules regarding the use of JI in the EU ETS from 2013 onwards led to this market mechanism also peaking supply in December 2012. Finally, the overallocation of emission allowances to economies in transition affected not only the market balance in the first commitment period of the Kyoto Protocol (2008-12) but is expected to keep permeating the market up to 2020.

For the CDM, this situation has led, in terms of numbers of projects, from a record of 320 projects being submitted for validation in a single month in April 2012 to a scant 18 projects in March 2013. It has furthermore resulted in the closure of carbon trading and project developer firms, in the loss of market experts into other business areas and in the renegotiation, termination or simple disregard of carbon purchase contracts with developing country firms (Point Carbon 2013a, 2013b). Trust in the $\mathrm{CDM}$, the EU ETS and carbon markets in general is rapidly decaying.

\subsection{THE CDM IN THE INTERNATIONAL CLIMATE NEGOTIATIONS}

Since its conception, the CDM has been a very innovative instrument in terms of its design, its institutions and its scope. It was hence bound to have deficiencies. Throughout its lifetime, a process of learning-by-doing has been experienced and a series of decisions have been taken by the COP and the Conference of the Parties acting as the Meeting of the Parties to the Kyoto Protocol (COP/MOP) each year in order to improve the CDM in its management and its substance. The most important substantial issues that have been addressed so far include: 
- The non-eligibility of policies as CDM projects, but the possibility to register programmes of activities as single projects.

- Whether new project categories, such as new HFCF-22 facilities, carbon capture and storage (CCS) and forests in exhaustion can be considered CDM projects.

- Improvements to the demonstration of additionality.

- Improvements to the governance of the CDM and the competences of all the involved bodies, including the work of DOEs, through, for example, an accreditation standard and a manual.

- Several measures to improve the geographical distribution of the CDM, such as the identification of barriers, the launch of the Nairobi Framework, the elimination of fees for projects in least developed countries (LDCs) and, more recently, the establishment of a loan scheme for CDM project development in underrepresented countries, as well as the development of top-down methodologies appropriate for their circumstances.

- The simplification of baseline methodologies, for example, through establishing simple methods for small-scale and micro-scale projects, and through the development of standardized baselines.

- The treatment of national policies that support emission reductions in relation to the CDM.

But negotiations about how to improve the CDM have also been embedded in a larger effort to agree on a new (or improved) climate change regime for the period after 2012. The entry into force of the Kyoto Protocol in 2005 and the recognition of the need to continue the efforts to combat climate change after the end of its first commitment period in 2012 led to renewed negotiations in the climate regime. In December 2005, the Ad-Hoc Working Group on Further Commitments for Annex I Parties under the Kyoto Protocol (AWG-KP) was created in order to decide upon new emission reduction targets for Annex I parties after 2012. In December 2007, within the process of periodic review of the Kyoto Protocol established in its Article 9, the COP/MOP decided that the second review shall consider, among other topics, ' $[\mathrm{t}]$ he scope, effectiveness and functioning of the flexibility mechanisms, including ways and means to enhance an equitable regional distribution of clean development mechanism projects' (UNFCCC 2008d, p. 19). Also in December 2007, the Bali Action Plan agreed at COP 13 created the Ad-Hoc Working Group on Long-term Cooperative Action under the Convention (AWG-LCA), which negotiates a new agreement that will enable the broader implementation of the convention.

On a more political level, in view of the challenges to the future 
operation and development of the CDM arising from the changing post2012 climate regime, a high-level CDM policy dialogue was launched in 2011. The goal of the CDM policy dialogue was to review past CDM experience and make recommendations on how to best position this instrument within future international climate policy. The dialogue was led by an independent panel of experts from all geographic regions and all stakeholder groups, was based on a broad consultation with civil society, market participants and policy makers, and published its final report in September 2012 (High-Level Panel on the CDM Policy Dialogue 2012). More recently, in December 2012, a call for inputs on possible changes to the CDM modalities and procedures was launched as part of the preparatory process towards the first review of the CDM modalities and procedures (UNFCCC 2012a). This review is planned to take place in COP/MOP 9 in December 2013, as decided in COP/MOP 1 in 2005.

The negotiations in these fora included further proposals on how to address the different shortcomings of the CDM described above, and on the role of the CDM and other market or non-market mechanisms in the post-2012 climate change regime. See, for example, UNFCCC (2008a, 2008e) for syntheses of proposals relating to the CDM made under the Subsidiary Body for Implementation and under the AWG-KP. While some of these proposals - particularly those related to reforming the CDM - are already being implemented, and others - especially those related to other mechanisms - are still under discussion, there has been very little empirical research on their expected effects on climate change mitigation in the South.

\subsection{THE CDM AND INCENTIVES FOR MITIGATION IN THE SOUTH}

This volume is thus motivated by the recognition that climate change mitigation needs to be strengthened, both in industrialized and developing countries. As the quotations at the beginning of this introductory chapter illustrate, addressing the causes of climate change requires ambitious action by all countries. Without denying that such efforts are to be led by industrialized countries through emission reduction targets that need to be much more ambitious than they are at present, the focus of the book is on incentives for action by developing countries.

In general terms, incentives for emission reduction action are similar for all countries: they will act if the benefits accruing from action are higher than the costs (Olson 1971). As will be described in Chapter 2, the public good nature of the climate makes such cooperation very difficult to 
achieve, both among industrialized and developing countries. Countries managed to start meaningful cooperation when they agreed to (albeit lenient) mandatory emission targets under the Kyoto Protocol in 1997. But the slow progress of negotiations on a new agreement and the substantially reduced scope of the Kyoto Protocol's mandatory framework after 2012 are clear signs of the cooperation dilemma at hand.

Assuming that cooperation is, in general terms, possible, this book looks at the effect of the design of the pre-2012 climate regime on future cooperation by developing countries, specifically at the role of the CDM in creating incentives - or disincentives - for developing country action towards reducing emissions. This implies action that will not only reduce the costs of mitigation elsewhere through offsetting but that will itself generate short- or long-term emission reductions. Academically, the book thus seeks to contribute to the literatures on international cooperation, environmental economics and public policy design. In practical terms, it expects to provide new insights in the analysis of proposals to reform or replace the CDM.

To do this, the book relies on three general assumptions about how the CDM may create positive incentives towards reducing emissions in developing countries: ${ }^{7}$

1. Positive incentives can be created if the CDM is successful in facilitating investment in low-carbon technologies in countries in which these technologies would otherwise be out of reach. Even if CDM credits are used for offsetting, the investments incentivized would generate a more climate-friendly long-term development path and prevent a lock-in to emissions-intensive technologies. I assume that this condition is applicable to LDCs, in which poverty alleviation concerns have a clear priority over global environmental goals.

2. In countries that are already able to afford less emissions-intensive technologies (advanced developing countries), ${ }^{8}$ the CDM can create positive incentives by setting the ground for a transition towards nonoffset mitigation instruments.

3. Both in advanced developing countries and in less developed countries, the CDM can incentivize mitigation if it promotes investment in low-carbon technologies that are not yet completely mature or commercially competitive, contributing to learning and scale effects that make these technologies more competitive in the long term.

Starting from these assumptions, I seek to address the gap in empirical research on the potential effects of the CDM and some of its reform proposals on climate change mitigation in the South, through an empirical assessment of: 
(a) Concrete measures that have been proposed to address barriers to its implementation in LDCs and make its geographical distribution more equitable.

(b) Potential perverse incentives that the CDM may generate in its major host countries.

(c) The effect of domestic climate-friendly policies in non-Annex I countries on investment in expensive emission reduction opportunities through the CDM.

Figure 1.2 graphically presents the structure of the book in relation to the assumptions and the research objectives listed above. Before proceeding to the empirical analysis, Chapter 2 details the conceptual framework that guides the study.

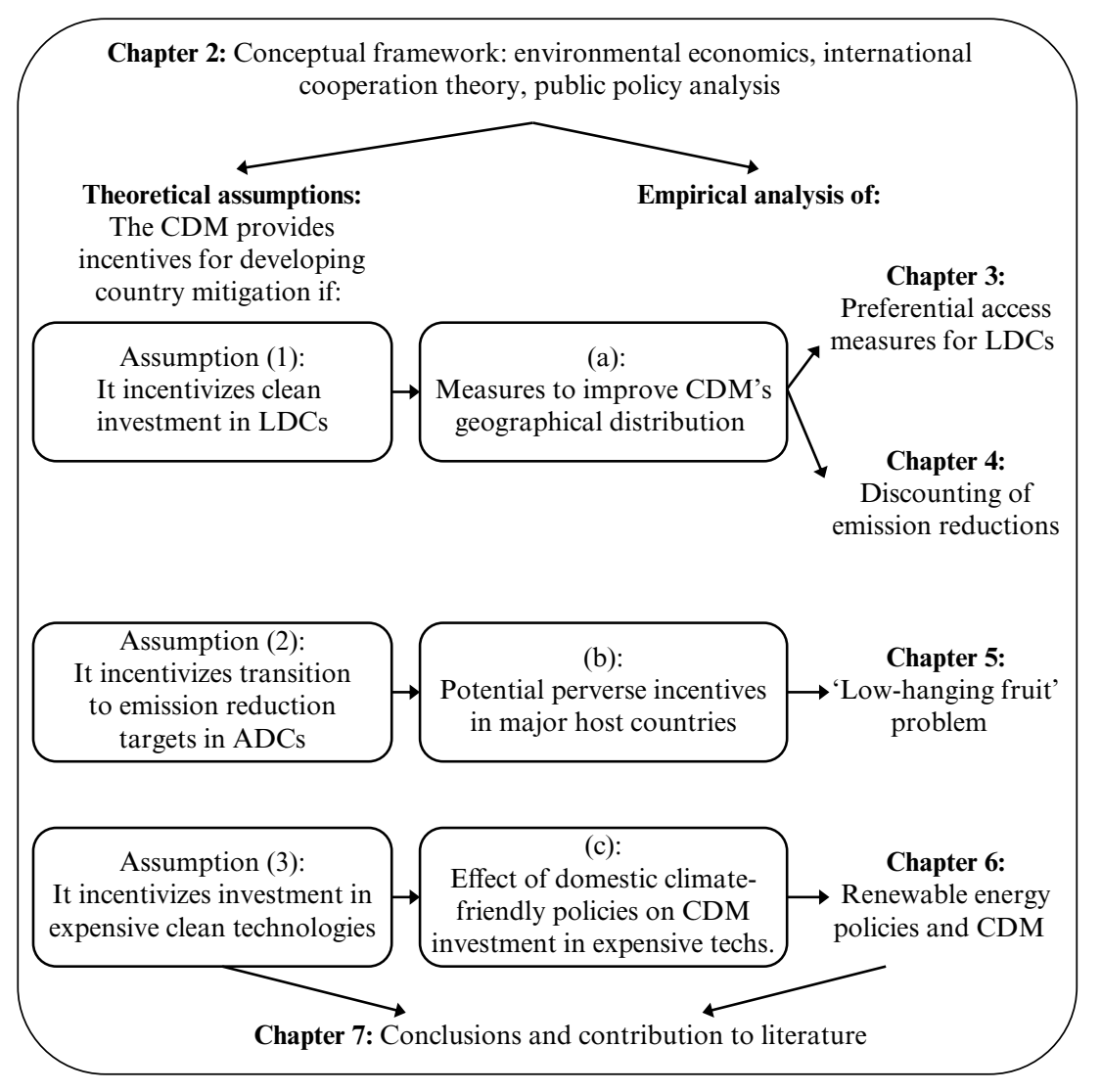

Figure 1.2 Structure of the book 
Chapters 3 and 4 address objective (a) described above by looking at measures proposed to generate incentives for increased CDM participation in LDCs and other poor countries with limited emission reduction potential and financial capacity. Such increased participation is desirable from two points of view. On the one hand, LDCs would benefit from more CDM projects because they would generate an inflow of financial resources, technology transfer and, per the CDM's objectives, sustainable development. On the other, while the climate change regime explicitly recognizes the limited capabilities of LDCs (UN 1992, Art. 4.9; Decision 5/CP.7 in UNFCCC 2001a, p. 33), so that they are not expected to undertake mitigation actions on their own, it is desirable that they should achieve growth in a more climate-friendly manner to avoid a lock-in to high-emitting technologies (assumption (1) above). The CDM, which was expected to contribute to this goal, has not been able to do so due to its limited presence in these countries.

The establishment of preferential access measures for CDM projects from LDCs is one of the measures that have been proposed to address the issue of the unequal geographical distribution of CDM projects. While the UNFCCC has granted financial support for projects in underrepresented CDM host countries (UNFCCC 2009), the EU has unilaterally introduced quality restrictions on the CDM projects that will be accepted in its carbon market from 2013 onwards. Among these restrictions is a clause establishing that in case no new international climate change agreement is reached, only CERs from LDCs, small island developing states (SIDSs) or countries with bilateral agreements with the EU will be accepted in the European ETS (European Parliament 2009b). So far, very little research has been carried out on whether such preferential access measures will have the desired effect of improving the geographic distribution of the CDM.

In Chapter 3, this question is addressed by assessing the impact of such preferential access measures on the carbon market through the comparison of several emission reduction credit supply and demand scenarios for the post-2012 period. The main methodological contribution of the chapter consists of a new approach for estimating future CER supply on the basis of data on the current CDM pipeline, which is more accurate than a simple aggregation of the existing figures as it incorporates the effects of failing to achieve registration at the different stages of the CDM project cycle. Furthermore, lessons for preferential treatment for LDCs under the CDM are drawn from a comparison with preferential access measures that have been implemented in the past in another international policy area: agriculture.

Chapter 4 analyses the introduction of discount factors for emission 
reductions achieved in advanced developing countries and its effect on the geographical distribution of the CDM, with particular focus on less developed countries and sub-Saharan Africa (SSA). Under discounting, each tonne of emission reductions achieved through the CDM would have a value of less than one tonne in the international carbon market. Depending on its concrete design, discounting the value of emission credits could be used to compensate for non-additional CDM projects; to increase the incentive for advanced developing countries to move from the CDM to own mitigation commitments (if the discount rates are set up to be more stringent for more advanced countries); and to improve the competitiveness of less developed countries as hosts for CDM projects (Chung 2007; Michaelowa 2008b; Schneider 2009). While there has been substantive discussion in the literature on how discounting of emission reductions could be implemented, and with what purposes it could be used, there has been little analysis on what would be its effects on the carbon market. Stoft (2009) makes a stylized analysis of the effects of discounting (and other proposed reforms of the CDM) on the basis of microeconomic theory, but does not look at implications for geographical distribution of projects. In Chapter 4, the impact of discounting on the competitiveness of CDM host countries is analysed by creating and comparing CDM-specific marginal abatement cost curves for four regions under two different discounting schemes. This chapter (as well as Chapter 5) relies on the creation of a new dataset of CDM projects' abatement costs, which were estimated on the basis of information provided in the projects' public documentation. The main methodological contribution of the chapter is thus the method for estimating these abatement costs in a credible and comparable manner, the compilation of the dataset, and the presentation and analysis of the information on the basis of marginal abatement cost curves, including the effect of discounting.

In Chapters 5 and 6, the focus is shifted towards incentives for advanced developing countries, which have been successful in the CDM, and which are under pressure to take on own mitigation actions beyond offsetting soon (objective (b) above). Some undesired perverse incentives that the CDM might generate for the countries that are benefiting the most from it have been identified early on. First, the financial transfers provided by the CDM to developing countries may make them unwilling to depart from the CDM to a more costly emission reduction target (Wara and Victor 2008; Burniaux et al. 2009; Hagem and Holtsmark 2009). Second, the need to demonstrate the additionality of CDM projects may discourage host country governments from adopting policies that may contribute to reduce emissions (Hepburn 2007). Third, even before the full implementation of the CDM, developing country experts feared that it would exhaust 
the cheap emission reduction opportunities in these countries, leaving them with only more expensive options to address eventual emission reduction targets of their own (World Bank 2003, p. 32; Narain and van't Veld 2008). Such concerns affect the negotiations about the future of the $\mathrm{CDM}$ and of other market mechanisms even today.

Chapter 5 thus focuses on analysing whether such a 'low-hanging fruits' problem has arisen in the current CDM. Theoretical economic models have been used to analyse under which conditions the low-hanging fruits problem would arise (for example, Akita 2003; Germain et al. 2007; Narain and van't Veld 2008). However, as these analyses were made before the CDM had started to work, there was no empirical evidence yet of the problem. Chapter 5 addresses this gap by looking empirically at the question of whether the CDM has so far exhausted many of the emission reduction opportunities existing in its most successful countries, using again marginal abatement cost curves for six of the countries that currently host most CDM projects, and relying on a larger, self-compiled dataset of CDM project abatement costs. The chapter thus seeks to contribute to the more general discussion on the role of offset mechanisms in achieving global GHG emission reductions.

Among other things, the abatement cost analysis in Chapter 5 reveals that there are some surprisingly expensive projects in the CDM. This appears puzzling from the point of view that rational economic actors would exhaust the cheap emissions abatement opportunities first, before investing in more expensive ones. It also seems to contradict the CDM critics' argument that its focus on cheap credits, coupled with its projectby-project nature and the low carbon price, is not contributing to a real systemic change in energy or industrial systems in developing countries. Chapter 6 investigates this phenomenon starting from the hypothesis that domestic-level financial incentives are contributing to finance relatively expensive CDM projects (objective (c) above). Using econometric techniques, this chapter thus analyses whether domestic policies providing financial incentives to climate-friendly projects - specifically renewable energy projects - in developing countries have an effect on the size of the CDM investment in renewable energy projects, after controlling for all other factors that may be influencing such investments as well. This chapter addresses several gaps in the literature. In general terms, I am not aware of any cross-country study that systematically analyses the factors leading to investment in renewable energies, especially in developing countries. While the chapter is focused on renewable energy investments within the CDM, it also controls for other drivers, and analyses in particular the effect of national policies. Furthermore, it combines insights from the general literature on investment in renewable energy with insights from 
the more specific literatures on barriers to CDM investment and factors leading to CDM investment, and with the literature on determinants of the adoption of environmentally friendly policies, in order to develop a theoretical framework for the empirical assessment. More specifically, within the literature on the CDM, the discussion about the interaction between national policies and the CDM incentive has been theoretical (for example, Hepburn 2007) or based on case studies (He and Morse 2010). This chapter provides first systematic empirical evidence that such an interaction exists.

Finally, Chapter 7 presents a summary of the conclusions in terms of whether the CDM has created incentives for developing country action towards mitigating climate change, and an outlook for the future climate regime in terms of how the limitations of the CDM could, to some extent, be addressed by new international instruments supporting mitigation in developing countries.

\section{NOTES}

1. Annex I of the UNFCCC lists the industrialized countries that were members of the Organisation for Economic Co-operation and Development (OECD) in 1992, plus countries with economies in transition, including the Russian Federation, the Baltic States and several Central and Eastern European states. Under the convention, these countries agreed to reduce GHG emissions to 1990 levels by the year 2000. Annex B of the Kyoto Protocol is an update of Annex I and lists those industrialized countries with mandatory emission reduction targets for the period 2008-12. Countries in both lists are the same, except for Belarus and Turkey that do not appear in Annex B, the United States (US), which has not ratified the Kyoto Protocol, and Canada, which withdrew in 2012. Following the commonly used terminology, throughout this book the terms 'industrialized countries' and 'Annex I countries' will be used indistinctly to denote those countries that currently have emission reduction commitments under the Kyoto Protocol. Countries without such emission reduction commitments will be denoted 'developing countries' or 'non-Annex I countries'. It should be noted, however, that this concept of developing countries also includes rapidly industrializing economies such as China, south-eastern Asian countries and others.

2. Due to the fact that the US did not ratify the Kyoto Protocol and Canada withdrew in December 2012, this overall target will likely not be met.

3. CERs are the trading currency of the CDM. One CER corresponds to one tonne of $\mathrm{CO}_{2}$-equivalent emission reductions. Once a project's reductions have been verified and certified by an independent auditor (so-called validator), it is issued the corresponding amount of CERs, which can be traded (or have been traded in advance) in the international carbon market.

4. A CDM project's baseline is the 'scenario that reasonably represents the anthropogenic emissions by sources of greenhouse gases that would occur in the absence of the proposed project activity' (UNFCCC 2005a, p. 16). It is thus the basis upon which the emission reductions accruing from a project are calculated.

5. A CDM project is said to be additional if 'anthropogenic emissions of greenhouse gases by sources are reduced below those that would have occurred in the absence of the registered CDM project activity' (UNFCCC 2005a, p. 16), that is, if it demonstrates that 
the situation without the project (baseline) is the most credible course of action in the absence of the CDM incentive. A more detailed discussion of the concept may be found in the introduction to Chapter 4 of this volume.

6. The CDM EB is the body that supervises the operation of the CDM, and is responsible, inter alia, for the accreditation of DOEs, the approval of baseline and monitoring methodologies, the provision of recommendations to the COP/MOP on further modalities and procedures of the $\mathrm{CDM}$, maintenance of a database of all projects and the provision of guidance to CDM projects. The CDM EB is fully accountable to the COP/MOP (UNFCCC 2005b). To fulfil these tasks, it is assisted by several advisory bodies, such as the Methodologies Panel, the Accreditation Panel, the Registration and Issuance Team, the Small-Scale Working Group, the Afforestation and Reforestation Working Group, as well as the UNFCCC Secretariat.

7. The theoretical rationale behind these assumptions will be explained in detail in Chapter 2.

8. For the purposes of this book, advanced developing countries are defined on the basis of the principle of common but differentiated responsibilities and respective capabilities, that is, in terms of the country's responsibility for causing climate change and capabilities for dealing with it. Under this rationale, countries with high GHG emission levels (high responsibility) and with high income per capita (high capability) are considered 'advanced'. I do not define a threshold for how high these indicators should be, as this is a political decision that will eventually need to be negotiated by the UNFCCC parties. However, several authors have proposed different ways of differentiating among developing countries in the climate regime (see, for example, Berk and den Elzen 2001; Ott et al. 2004, pp. 26ff; Michaelowa et al. 2005; Karousakis et al. 2008; Bakker et al. 2009).

In addition, the term 'less developed countries' refers to all those developing countries that have a lower level of responsibility and capability to respond to climate change than the advanced developing countries, and should therefore not be considered equivalent to the more specific group of the LDCs (least developed countries). 